\title{
Two open problems on effective dimension
}

\author{
Elvira Mayordomo \\ Dept. de Informática e Ing. de Sistemas, Universidad de Zaragoza, \\ María de Luna 1, 50018 Zaragoza, SPAIN. \\ elvira@unizar.es
}

\section{Introduction}

Effective fractal dimension was defined by Lutz [13] in order to quantitatively analyze the structure of complexity classes. The dimension of a class $X$ inside a base class $\mathcal{C}$ is a real number in $[0,1]$ corresponding to the relative size of $X \cap \mathcal{C}$ inside $\mathcal{C}$. Basic properties include monotonicity, so dimension 1 classes are maximal and dimension 0 ones are minimal, and the fact that dimension is defined for every class $X$, making effective dimension a precise quantitative tool.

The first goal of such quantitative methods is to extend existence results of the form "there is a problem in $\mathcal{C}$ that is in $X$ " to abundance results of the form "a non-negligible part of the problems in $\mathcal{C}$ are in $X$ " formally expressed as "the class $X$ has positive dimension in $\mathcal{C}$ ". Another application is in relation with the probabilistic method, proving that $X$ has positive dimension can be simpler than proving non-emptiness, the easiness here comes from proving abundance as opposed to constructing a particular object. A third aspect of effective dimension is as a formal tool in Computational Complexity, allowing us to consider new working hypothesis such as "NP has positive dimension in exponential time", that can imply plausible consequences that haven't been derived from $\mathrm{P} \neq \mathrm{NP}$.

The concept of effective dimension is a generalization of classical fractal or Hausdorff dimension, one of the most powerful tools of fractal geometry, an extensively developed subfield of geometric measure theory with applications throughout the sciences $[5,7,6]$. Tricot [19] and Sullivan [18] independently developed a dual of Hausdorff dimension called packing dimension, that now rivals Hausdorff dimension's importance in such investigations.

In 2000 Lutz proved a new characterization of Hausdorff dimension in terms of gales [13], that are betting strategies that generalize martingales. The most important benefit of this characterization is that it enables one to define effective versions of fractal dimension by imposing various computability and complexity constraints on the gales. Four years later Athreya, Lutz, Hitchcock and Mayordomo [3] proved that packing dimension also admits a gale characterization, with a different notion of gale success. We can now define versions of Hausdorff and packing dimensions that are meaningful inside complexity classes such as exponential time and exponential space.

\footnotetext{
* This research was supported in part by Spanish Government MEC project TIN 2005-08832-C03-02
} 
Effective dimension has indeed proven to be very fruitful in Computational Complexity for obtaining useful results in the three aspects mentioned above. A very recent summary of the main achievements can be found in [10].

In this note we propose two interesting open problems on Computational Complexity, both related to polynomial-time reductions. Complete or partial solutions of these problems imply a big advance in what we know on the classes NP and BPP. In both cases quantitative methods such as resource-bounded measure have given initial answers in the past, and the fact that dimension is defined for every class can overcome non-measurability obstacles.

\section{Effective dimension}

For the sake of completeness we include the basic definitions of resource-bounded or effective dimension, based on the notion of $s$-gale. A more detailed treatment, motivation, references and historical introduction can be found in [10], [15], and [14].

We work in the Cantor space $\mathbf{C}$ that is the set of all infinite binary sequences. $\{0,1\}^{*}$ is the set of finite binary strings.

Formally, if $s \in[0, \infty)$, then an $s$-gale is a function $d:\{0,1\}^{*} \rightarrow[0, \infty)$ satisfying the condition

$$
d(w)=2^{-s}[d(w 0)+d(w 1)]
$$

for all $w \in\{0,1\}^{*}[13]$. A martingale is a 1-gale.

A gale $d$ succeeds on a sequence $S$ if

$$
\limsup _{w \rightarrow S} d(w)=\infty
$$

and succeeds strongly on $S$ if

$$
\liminf _{w \rightarrow S} d(w)=\infty
$$

The success set $S^{\infty}[d]$ of a gale $d$ is the set of all sequences on which $d$ succeeds. The strong success set $S_{\mathrm{str}}^{\infty}[d]$ is the set of all sequences on which $d$ succeeds strongly.

Intuitively, we think of a gale $d$ as a strategy for betting on the successive bits of a sequence $S$. The quantity $d(w)$ is interpreted as the capital (amount of money) that a gambler using the strategy $d$ has after betting on the successive bits of the prefix $w$ of $S$. The parameter $s$ regulates the fairness of the payoffs via identity (1). If $s=1$, the payoffs are fair in the usual sense that the conditional expectation of the gambler's capital $d(w b)$, given that $w$ has occurred, is precisely $d(w)$, the gambler's actual capital before betting on the last bit of $w b$. If $s<1$, then the payoffs are unfair, and the smaller $s$ is, the more unfair the payoffs are.

Theorem 1. (Gale characterization of fractal dimension) Let $X$ be a set of sequences. 
1. (Lutz [13]) $\operatorname{dim}_{\mathrm{H}}(X)=\inf \left\{s \mid\right.$ there is an s-gale $d$ such that $\left.X \subseteq S^{\infty}[d]\right\}$.

2. (Athreya et al. [3]) $\operatorname{dim}_{\mathrm{P}}(X)=\inf \{s \mid$ there is an s-gale $d$ such that $\left.X \subseteq S_{\mathrm{str}}^{\infty}[d]\right\}$.

Intuitively, Theorem 1 says that the fractal dimension of a set $X$ of sequences is the most hostile environment (i.e., most unfair payoff parameter $s$ ) in which a gambler can win on every sequence in $X$. Of course, the word "win" here means "succeed" in the case of Hausdorff dimension and "succeed strongly" in the case of packing dimension.

It is easy to see that $0 \leq \operatorname{dim}_{\mathrm{H}}(X) \leq \operatorname{dim}_{\mathrm{P}}(X) \leq 1$ holds in any case. Both of these fractal dimensions are monotone (i.e., $X \subseteq Y$ implies $\operatorname{dim}(X) \leq$ $\operatorname{dim}(Y))$, countably stable (i.e., $\left.\operatorname{dim}\left(\bigcup_{i=0}^{\infty} X_{i}\right)=\sup _{i} \operatorname{dim}\left(X_{i}\right)\right)$, and nonatomic (i.e., $\operatorname{dim}(\{S\})=0$ for each sequence $S$ ) [6]. In particular, every countable set of sequences has Hausdorff and packing dimension 0.

We say that a gale $d:\{0,1\}^{*} \rightarrow[0, \infty)$ is p-computable if there is a function $\hat{d}:\{0,1\}^{*} \times \mathbb{N} \rightarrow \mathbb{Q}$ such that $\hat{d}(w, r)$ is computable in time polynomial in $|w|+r$ and $|\hat{d}(w, r)-d(w)| \leq 2^{-r}$ holds for all $w$ and $r$. Gales that are $\mathrm{p}_{2}$-computable are defined analogously, with $\hat{d}(w, r)$ required to be computable in $2^{(\log (|w|+r))^{O(1)}}$ time.

We are finally ready to bring this all home to complexity classes. We identify each language (i.e., decision problem) $A \subseteq\{0,1\}^{*}$ with its characteristic sequence, whose $n^{\text {th }}$ bit is 1 if the $n^{\text {th }}$ string in $\{0,1\}^{*}$ (in the standard enumeration $\lambda, 0,1,00,01, \ldots)$ is an element of $A$, and 0 otherwise. We say that a gale succeeds on $A$ if it succeeds on the characteristic sequence of $A$ and similarly for strong success. We now show how to define fractal dimension in the complexity classes $\mathrm{E}=\operatorname{TIME}\left(2^{\text {linear }}\right)$ and $\operatorname{EXP}=\operatorname{TIME}\left(2^{\text {polynomial }}\right)$.

Definition 1. $[13,3]$ Let $X$ be a set of languages.

1. If $\Delta$ is any of the resource bounds $\mathrm{p}, \mathrm{p}_{2}$, then the $\Delta$-dimension of $X$ is

$\operatorname{dim}_{\Delta}(X)=\inf \left\{s \mid\right.$ there is a $\Delta$-computable s-gale $d$ such that $\left.X \subseteq S^{\infty}[d]\right\}$, and the strong $\Delta$-dimension of $X$ is

$\operatorname{Dim}_{\Delta}(X)=\inf \left\{s \mid\right.$ there is a $\Delta$-computable s-gale d such that $\left.X \subseteq S_{\mathrm{str}}^{\infty}[d]\right\}$.

2. The dimension of $X$ in $\mathrm{E}$ is $\operatorname{dim}(X \mid \mathrm{E})=\operatorname{dim}_{\mathrm{p}}(X \cap \mathrm{E})$.

3. The dimension of $X$ in EXP is $\operatorname{dim}(X \mid \mathrm{EXP})=\operatorname{dim}_{\mathrm{p}_{2}}(X \cap \mathrm{EXP})$.

4. The strong dimensions $\operatorname{Dim}(X \mid \mathrm{E}), \operatorname{Dim}(X \mid \mathrm{EXP})$ are defined analogously.

By Theorem $1, \operatorname{dim}(X \mid \mathcal{C})$ and $\operatorname{Dim}(X \mid \mathcal{C})$ are analogs of Hausdorff and packing dimension, respectively. It was shown in $[13,3]$ that these analogs are in fact well-behaved, internal dimensions in the classes $\mathcal{C}$ that we have mentioned. In all these classes, $0 \leq \operatorname{dim}(X \mid \mathcal{C}) \leq \operatorname{Dim}(X \mid \mathcal{C}) \leq 1$ hold, with $\operatorname{dim}(\mathcal{C} \mid \mathcal{C})=1$. 


\subsection{Scaled dimension}

Scaled dimension [9] are versions of resource-bounded dimension that have been "rescaled" to better fit the phenomena that they are measuring. They correspond to the concept of generalized dimension already suggested by Hausdorff.

Scaled dimension arises using more general factors than $2^{-s}$ in the definition of gale in equation 1. In the general theory, there is a natural hierarchy of scales $g_{i}(s, n)$, one for each integer $i \in \mathbb{Z}$, built around the standard scale

$$
g_{0}(m, s)=m s .
$$

The $i^{\text {th }}$ scale gives us $i^{\text {th }}$-order scaled dimension.

The first scales are the following, for $0 \leq s \leq 1$,

$$
\begin{aligned}
& g_{3}(m, s)=2^{2^{(\log \log m)^{s}}} \\
& g_{2}(m, s)=2^{(\log m)^{s}} \\
& g_{1}(m, s)=m^{s} \\
& g_{0}(m, s)=m s \\
& g_{-1}(m, s)=m+1-m^{1-s} \\
& g_{-2}(m, s)=m+2-2^{(\log m)^{1-s}} \\
& g_{-3}(m, s)=m+2^{2}-2^{2^{(\log \log m)^{1-s}}}
\end{aligned}
$$

We refer to [9] for a justification of the choice of these scales, related for instance to complexity classes such as $\operatorname{SIZE}\left(2^{n \alpha}\right)$ and $\operatorname{SIZE}\left(2^{n^{\alpha}}\right)$.

An $s^{(i)}$-gale is a function $d:\{0,1\}^{*} \rightarrow[0, \infty)$ satisfying

$$
d(w)=2^{-g_{i}(|w|+1, s)+g_{i}(|w|, s)}[d(w 0)+d(w 1)]
$$

for all $w \in\{0,1\}^{*}$.

The concept of success and strong success of an $s^{(k)}$-gale on a sequence is defined exactly as in the previous section, the corresponding limsup (liminf) must be infinity.

Definition 2. [9] Let $X$ be a set of languages.

1. If $\Delta$ is any of the resource bounds $\mathrm{p}, \mathrm{p}_{2}$, then the $i^{\text {th }}$-order scaled $\Delta$-dimension of $X$ is

$$
\begin{gathered}
\operatorname{dim}_{\Delta}^{(i)}(X)=\inf \left\{s \mid \text { there is a } \Delta \text {-computable } s^{(i)}\right. \text {-gale d such that } \\
\left.X \subseteq S^{\infty}[d]\right\} .
\end{gathered}
$$

2. The $i^{\text {th }}$-order scaled dimension of $X$ in $\mathrm{E}$ is $\operatorname{dim}^{(i)}(X \mid \mathrm{E})=\operatorname{dim}_{\mathrm{p}}^{(i)}(X \cap \mathrm{E})$.

3. The $i^{\text {th }}$-order scaled dimension of $X$ in EXP is $\operatorname{dim}^{(i)}(X \mid \operatorname{EXP})=\operatorname{dim}_{\mathrm{P}_{2}}^{(i)}(X \cap$ EXP). 
The $i^{\text {th }}$-order scaled strong $\Delta$-dimension of $X$, written $\operatorname{Dim}_{\Delta}^{(i)}(X)$, is defined in the same way, instead requiring strong success of the scaled-gale. We also define $\operatorname{Dim}^{(i)}(X \mid \mathrm{E})=\operatorname{Dim}_{\mathrm{p}}^{(i)}(X \cap \mathrm{E}), \operatorname{Dim}^{(i)}(X \mid \mathrm{EXP})=\operatorname{Dim}_{\mathrm{p}_{2}}^{(i)}(X \cap \mathrm{EXP})$, etc. (analogously to the definitions in Section 2).

The $0^{\text {th }}$-order scaled dimension is the standard (unscaled) dimension. The other scaled dimensions have similar properties. For example, $0 \leq \operatorname{dim}_{\Delta}^{(i)}(X) \leq$ $\operatorname{Dim}_{\Delta}^{(i)}(X) \leq 1$ and if $\operatorname{dim}_{\Delta}^{(i)}(X)<1$, then $X$ has $\Delta$-measure 0 . The following theorem states two important facts about the scaled dimensions.

Theorem 2. [9] The scaled dimension $\operatorname{dim}_{\Delta}^{(i)}(X)$ is nondecreasing in the order $i$. There is at most one order $i$ for which $\operatorname{dim}_{\Delta}^{(i)}(X)$ is not 0 or 1 .

In particular, the sequence of scaled dimensions must have one of the following four forms.

(i) For all $i, \operatorname{dim}_{\Delta}^{(i)}(X)=0$.

(ii) For all $i, \operatorname{dim}_{\Delta}^{(i)}(X)=1$.

\begin{tabular}{|c|c|}
\hline There is an order $i^{*}$ such that \\
(iii) $-\operatorname{dim}_{\Delta}^{(i)}(X)=0$ for all $i \leq i^{*}$ and \\
$-\operatorname{dim}_{\Delta}^{(i)}(X)=1$ for all $i>i^{*}$.
\end{tabular} \mid $\begin{aligned} & \text { There is an order } i^{*} \text { such that } \\
& -\operatorname{dim}_{\Delta}^{(i)}(X)=0 \text { for all } i<i^{*}, \\
& -0<\operatorname{dim}_{\Delta}^{\left(i^{*}\right)}(X)<1 \text {, and } \\
& -\operatorname{dim}_{\Delta}^{(i)}(X)=1 \text { for all } i>i^{*} .\end{aligned}$

We find (iv) to be the most interesting case. Then $i^{*}$ is the "best" order at which to measure the $\Delta$-dimension of $X$ because $\operatorname{dim}_{\Delta}^{\left(i^{*}\right)}(X)$ provides much more quantitative information about $X$ than is provided by $\operatorname{dim}_{\Delta}^{(i)}(X)$ for $i \neq i^{*}$.

\section{Small span theorems and BPP}

We classify an apparently intractable problem $A$ by identifying and studying the class of all problems that are efficiently reducible to $A$. Efficiently reducible can be taken as polynomial-time many-one reducible $\left(\leq_{\mathrm{m}}^{\mathrm{P}}\right.$-reductions $)$, polynomialtime Turing reducible $\left(\leq_{\mathrm{T}}^{\mathrm{P}}\right.$-reductions) or any of the intermediate reductions obtained by restricting the query mechanism in polynomial-time Turing reducibilities.

The lower $\leq_{\mathrm{m}}^{\mathrm{P}}$-span of $A$ is the set of problems that are $\leq_{\mathrm{m}}^{\mathrm{P}}$-reducible to $A$

$$
\mathrm{P}_{\mathrm{m}}(A)=\left\{B \mid B \leq_{\mathrm{m}}^{\mathrm{P}} A\right\}
$$

and similarly for other reductions, and the upper $\leq_{\mathrm{m}}^{\mathrm{P}}$-span is the set $\mathrm{P}_{\mathrm{m}}^{-1}(A)$ consisting of those decision problems $B$ to which $A$ is $\leq_{\mathrm{m}}^{\mathrm{P}}$-reducible. 
A Small Span Theorem for a reduction $\leq_{\mathrm{r}}^{\mathrm{P}}$ in a class $\mathcal{C}$ is the assertion that for every $A \in \mathcal{C}$ it must be the case that either $\mathrm{P}_{r}(A)$ or $\mathrm{P}_{\mathrm{r}}^{-1}(A)$ have minimal size. This kind of result implies that the degree structure of $\mathcal{C}$ is very fine, so whenever a subclass is not minimal it must contain problems in several degrees.

Juedes and Lutz [12] obtained the first Small Span Theorem for the reduction $\leq_{\mathrm{m}}^{\mathrm{P}}$ and both classes E and EXP, using resource-bounded measure. Other authors pushed this result to $\leq_{\mathrm{k}-\mathrm{tt}}^{\mathrm{P}}$ in $\mathrm{E}$ and $\leq_{n^{\circ(1)}}^{\mathrm{P}}$ in $\operatorname{EXP}[2,4]$.

In dimension the situation is far more complicated, sice Ambos-Spies et al. [1] and later Hitchcock [8] proved that for scales $i \geq-2$ there are degrees of maximal p-dimension 1. For scale -3 , Hitchcock [8] proved a Small Span Theorem for $\leq_{\mathrm{m}}^{\mathrm{P}}$ and E.

An important application of Small Span Theorems is related to BPP, the class corresponding to probabilistic polynomial time, since the class of hard problems for BPP has maximal size in all quantitative settings (Martin-Löf tests, resourcebounded measure, effective dimensions) also when restricted to the subclass of hard sets for BPP in EXP. The existence of a Small Span Theorem for $\leq_{\mathrm{T}}^{\mathrm{P}}$ or $\leq_{\mathrm{tt}}^{\mathrm{P}}$ would imply the separation of BPP and EXP, since the degree of Turingcomplete sets for EXP would then be minimal size.

Open question. Prove that for every $A \in \mathrm{EXP}$

$$
\operatorname{dim}^{(-3)}\left(\mathrm{P}_{\mathrm{T}}(A) \mid \mathrm{EXP}\right)=0 \quad \text { or } \quad \operatorname{dim}^{(-3)}\left(\mathrm{P}_{\mathrm{T}}^{-1}(A) \mid \mathrm{EXP}\right)
$$

Alternatively prove that $\operatorname{dim}^{(-3)}\left(\operatorname{deg}_{\mathrm{T}}^{\mathrm{P}}(A) \mid \mathrm{EXP}\right)=0$. Similar questions for $\mathrm{E}$ in the place of EXP and for $\leq_{\mathrm{tt}}^{\mathrm{P}}$ in the place of $\leq_{\mathrm{T}}^{\mathrm{P}}$ are relevant.

Notice that a solution to this question would be the least exigent form of a Small Span Theorem for dimension and polynomial-time reductions. Any statement about other scaled or strong dimensions is either false or would imply an affirmative answer to this. It is also weaker, thus an affirmative answer is more plausible, than a resource-bounded measure version.

\section{Completeness separations}

Even if we assume that $\mathrm{P} \neq \mathrm{NP}$, many open questions in Computational Complexity remain open. Lutz [17] proposed investigation of various strong, measuretheoretic hypothesis, the most notable of which is the hypothesis that NP does not have resource-bounded measure 0 in EXP, that is known now to have many interesting consequences (not known to follow from $\mathrm{P} \neq \mathrm{NP}$ ).

One of these consequences is the separation of the notions of $\leq_{\mathrm{m}}^{\mathrm{P}}$ and $\leq_{\mathrm{T}}^{\mathrm{P}}$ completeness for the class NP [16], a statement that seems far stronger than $\mathrm{P}$ versus NP.

The answer to each of the following questions would be an improvement over the result in [16].

Open questions. Prove that $\operatorname{dim}_{\mathrm{p}}(N P)>0$ implies the separation of $\leq_{\mathrm{T}}^{\mathrm{P}}$ and $\leq_{\mathrm{tt}}^{\mathrm{P}}$ completeness notions for NP.

Hitchcok et al. have proven in [11] that the separation of $\leq_{\mathrm{m}}^{\mathrm{P}}$ and $\leq_{\mathrm{T}^{-}}^{\mathrm{P}}$ completeness for NP can be obtained from the hypothesis $\operatorname{dim}_{\mathrm{p}}^{(-3)}(N P)>0$, 
which is weaker than the original measure hypothesis. Can this be improved to a bigger scale $(-2,-1,0, \ldots)$ ?

\section{References}

1. K. Ambos-Spies, W. Merkle, J. Reimann, and F. Stephan. Hausdorff dimension in exponential time. In Proceedings of the 16th IEEE Conference on Computational Complexity, pages 210-217, 2001.

2. K. Ambos-Spies, H.-C. Neis, and S. A. Terwijn. Genericity and measure for exponential time. Theoretical Computer Science, 168(1):3-19, 1996.

3. K. B. Athreya, J. M. Hitchcock, J. H. Lutz, and E. Mayordomo. Effective strong dimension in algorithmic information and computational complexity. SIAM Journal on Computing. To appear.

4. H. Buhrman and D. van Melkebeek. Hard sets are hard to find. Journal of Computer and System Sciences, 59(2):327-345, 1999.

5. K. Falconer. The Geometry of Fractal Sets. Cambridge University Press, 1985.

6. K. Falconer. Fractal Geometry: Mathematical Foundations and Applications. John Wiley \& sons, 2003.

7. K. Falconer. Techniques in Fractal Geometry. John Wiley \& sons, 2003.

8. J. M. Hitchcock. Small spans in scaled dimension. SIAM Journal on Computing, 34:170-194, 2004.

9. J. M. Hitchcock, J. H. Lutz, and E. Mayordomo. Scaled dimension and nonuniform complexity. Journal of Computer and System Sciences, 69:97-122, 2004.

10. J. M. Hitchcock, J. H. Lutz, and E. Mayordomo. The fractal geometry of complexity classes. SIGACT News Complexity Theory Column, 36:24-38, 2005.

11. J. M. Hitchcock, A. Pavan, and N. V. Vinodchandran. Partial bi-immunity, scaled dimension, and NP-completeness. Theory of Computing Systems. To appear.

12. D. W. Juedes and J. H. Lutz. The complexity and distribution of hard problems. SIAM Journal on Computing, 24(2):279-295, 1995.

13. J. H. Lutz. Dimension in complexity classes. SIAM Journal on Computing, $32: 1236-1259,2003$

14. J. H. Lutz. The dimensions of individual strings and sequences. Information and Computation, 187:49-79, 2003.

15. J. H. Lutz. Effective fractal dimensions. Mathematical Logic Quarterly, 51:62-72, 2005.

16. J. H. Lutz and E. Mayordomo. Cook versus Karp-Levin: Separating completeness notions if NP is not small. Theoretical Computer Science, 164(1-2):141-163, 1996.

17. J. H. Lutz and E. Mayordomo. Twelve problems in resource-bounded measure. In G. Paun, G. Rozenberg, and A. Salomaa, editors, Current Trends in Theoretical Computer Science: Entering the 21st Century, pages 83-101. World Scientific, 2001.

18. D. Sullivan. Entropy, Hausdorff measures old and new, and limit sets of geometrically finite Kleinian groups. Acta Mathematica, 153:259-277, 1984.

19. C. Tricot. Two definitions of fractional dimension. Mathematical Proceedings of the Cambridge Philosophical Society, 91:57-74, 1982. 